\title{
The Needlet CMB Trispectrum
}

\section{Antonino Troja ${ }^{1, \dagger}$, Simona Donzelli $^{2}$, Davide Maino $^{1}$ and Domenico Marinucci $^{3}$}

\author{
${ }^{1}$ Dipartimento di Fisica, Università degli Studi di Milano, \\ Via Celoria 16, 20133, Milano (MI), Italy \\ ${ }^{\dagger}$ email: antonino.troja@unimi.it \\ ${ }^{2}$ INAF - Istituto di Astrofisica Spaziale e Fisica Cosmica, Milano, \\ Via E. Bassini 15, 20133, Milano (MI), Italy \\ ${ }^{3}$ Dipartimento di Matematica, Università degli Studi di Roma Tor Vergata, \\ Via della Ricerca Scientifica, 00133, Roma (RM), Italy
}

\begin{abstract}
We propose a computationally feasible estimator for the needlet trispectrum, which develops earlier work on the bispectrum by Donzelli et al. (2012). Our proposal seems to enjoy a number of useful properties, in particular a) the construction exploits the localization properties of the needlet system, and hence it automatically handles masked regions; b) the procedure incorporates a quadratic correction term to correct for the presence of instrumental noise and sky-cuts; c) it is possible to provide analytic results on its statistical properties, which can serve as a guidance for simulations. The needlet trispectrum we present here provides the natural building blocks for the efficient estimation of nonlinearity parameters on CMB data, and in particular for the third order constants $g_{N L}$ and $\tau_{N L}$.
\end{abstract}

Keywords. cosmic microwave background, early universe

\section{Introduction}

The Inflationary models describe the dynamics of the first instants of the Universe. Each model introduces a characteristic signature of non-Gaussianity into the anisotropy distribution of the Cosmic Microwave Background (CMB) radiation. The level of nonGaussianity is described by the so-called Bardeen's Potential (Bardeen (1980)):

$$
\Phi(\mathbf{x})=\Phi_{L}(\mathbf{x})+f_{N L}\left[\Phi_{L}^{2}(\mathbf{x})-\left\langle\Phi_{L}^{2}(\mathbf{x})\right\rangle\right]+g_{N L}\left[\Phi_{L}^{3}(\mathbf{x})\right]
$$

where $\Phi(\mathbf{x})$ is the gravitational potential field of the Universe and $\Phi_{L}(\mathbf{x})$ is its Gaussian part. The constants $f_{N L}$ and $g_{N L}$, which parametrize the non-Gaussian part, can be measured from the amplitude of the bispectrum and trispectrum of the field, i.e. the harmonic counterpart of the 3-point and 4-point correlation function of the field, respectively.

There exist several optimal bispectrum estimators that allow to evaluate $f_{N L}$ with high confidence (see for instance Komatsu et al. (2005) or PLANCK Coll. (2013)). Optimal trispectrum estimators has been already applied to CMB data at WMAP resolution (e.g. Sekiguchi \& Sugiyama (2013) in real space and Regan et al. (2013) in needlet space), but the lack of an optimal trispectrum estimator applied to high-resolution sky simulations (e.g. PLANCK resolution) has prevented the evaluation of strong constraints on $g_{N L}$ so far. 


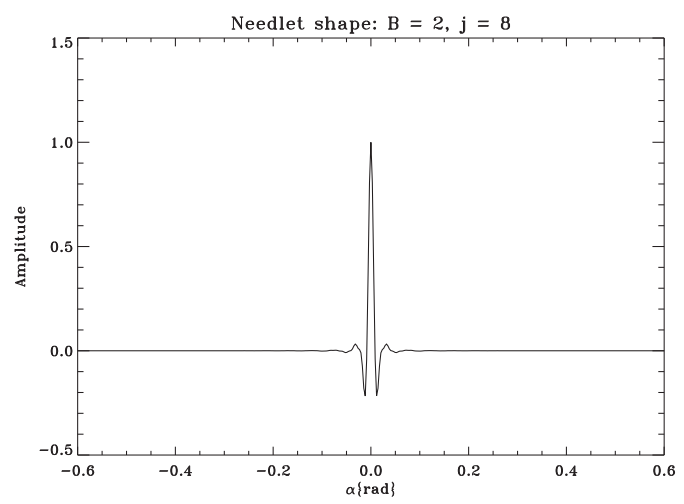

Figure 1. 1D-Spherical needlet. The figure shows the characteristic needle shape of the function, from which take its name (from Marinucci et al. (2008))

\section{Spherical Needlet System}

\subsection{Definition}

Spherical needlets (Narcowich et al. (2006), Baldi et al. (2009)) are a wavelet system on the sphere defined by setting:

$$
\psi_{j k}(x):=\sqrt{\lambda_{j k}} \sum_{l} b\left(\frac{l}{B^{j}}\right) \sum_{m=-l}^{l} Y_{l m}\left(\xi_{j k}\right) \overline{Y_{l m}}(x) ;
$$

where $x \in S^{2},\left\{\lambda_{j k}, \xi_{j k}\right\}$ are a set of cubature points and weights on the sphere, $B>1$ is a constant related to the width of the needlet and $b($.$) is a weight function satisfying the$ three following conditions: a) Compact Support, $b(\xi)>0$ if $\xi \in\left(B^{-1}, B\right), 0$ otherwise; b) Partition of Unity, for all $\zeta \geqslant 1, \sum_{j=0}^{\infty} b^{2}\left(\frac{\xi}{B^{j}}\right)=1$; c) Smoothness, $b(.) \in C^{M}$, i.e., $b($.$) is M$ times continuously differentiable, for some $M=1,2, \ldots$ or $M=\infty$.

\subsection{Reconstruction Formula}

The spherical needlet coefficients are provided by the analytical formula:

$$
\beta_{j k}=\int_{S^{2}} T(x) \psi_{j k}(x) d \sigma(x)=\sqrt{\lambda_{j k}} \sum_{l} b\left(\frac{l}{B^{j}}\right) \sum_{m=-l}^{l} a_{l m} Y_{l m}\left(\xi_{j k}\right) .
$$

As a consequence of the partition of unity property, the following well-known reconstruction formula holds:

$$
f(x)=\sum_{j k} \beta_{j k} \psi_{j k}(x) \equiv \sum_{l m} a_{l m} Y_{l m}(x) .
$$

\subsection{Localization and Uncorrelation Properties}

As argued earlier in Baldi et al. (2009), spherical needlets enjoy an excellent localization property in real domain (fig. 1):

$$
\left|\psi_{j k}(x)\right| \leqslant \frac{c_{M} B^{j}}{\left(1+B^{j} \arccos \left\langle\xi_{j k}, x\right\rangle\right)^{M}} .
$$

More explicitly, spherical needlets are then quasi-exponentially localized around any cubature point $\xi_{j k}$. Moreover, as extensively argued in the literature, the needlet coefficients 
evaluated on isotropic random fields are asymptotically uncorrelated, under mild regularity conditions. It is then possible to derive analytically their statistical properties, and to understand the role and expressions for correction terms under realistic experimental conditions (noise and masks, see Donzelli et al. for the bispectrum case)

\section{Optimal Trispectrum Estimator}

The sample trispectrum estimator is usually written using the spherical harmonics coefficients as:

$$
\sum_{m_{1} m_{2} m_{3} m_{4}} \mathcal{G}\left(l_{1}, m_{1}, l_{2}, m_{2}, l_{3}, m_{3}, l_{4}, m_{4}\right) \times a_{l_{1} m_{1}} a_{l_{2} m_{2}} a_{l_{3} m_{3}} a_{l_{4} m_{4}},
$$

where $\mathcal{G}\left(l_{1}, m_{1}, l_{2}, m_{2}, l_{3}, m_{3}, l_{4}, m_{4}\right)$ is the Gaunt integral defined for instance in Marinucci \& Peccati (2011). This estimator is clearly unfeasible under realistic experimental conditions, due to the presence of missing data and anisotropic noise. As a consequence of the previous discussion, it is possible to exploit the needlet coefficients to derive an alternative, computationally feasible and statistically sound estimators of the trispectrum. More precisely, exploiting Wick theorem on higher order moments of Gaussian variables we propose the following needlet trispectrum:

$$
\begin{aligned}
J_{j_{3} j_{4}}^{j_{1} j_{2}}= & \frac{1}{\sigma_{j_{1}} \sigma_{j_{2}} \sigma_{j_{3}} \sigma_{j_{4}}} \sum_{k}\left[\beta_{j_{1} k} \beta_{j_{2} k} \beta_{j_{3} k} \beta_{j_{4} k}\right. \\
& \left.-\left\{\left\langle\beta_{j_{1} k} \beta_{j_{2} k}\right\rangle \beta_{j_{3} k} \beta_{j_{4} k}+5 \text { perms. }\right\}+\left\{\left\langle\beta_{j_{1} k} \beta_{j_{2} k}\right\rangle\left\langle\beta_{j_{3} k} \beta_{j_{4} k}\right\rangle+2 \text { perms. }\right\}\right]
\end{aligned}
$$

Heuristically, the needlet trispectrum is constructed combining quadruples of coefficients, evaluated at the scales of interest, and subtracting linear and quadratic components in order to cancel bias and minimize the variance. Further details, and a software which exploits the needlet trispectrum to evaluate $g_{N L}$ on CMB maps, will be provided in the forthcoming paper Troja et al. (2014) .

\section{References}

Baldi, P., Kerkyacharian, G. Marinucci, D., \& Picard, D. 2009, Annals of Statistics, 37, 1150

Bardeen, J. M. 1980, Phys. Rev. D, 22, 1882

Donzelli, S., Hansen, F. K., Liguori, M., Marinucci, D., \& Matarrese, S. Apj

Komatsu, E., Spergel, D. N., \& Wandelt, B. D. 2005, ApJ, 634, 14

Marinucci, D. \& Peccati, G. 2011, Random Fields on the Sphere, Cambridge University Press

Marinucci, D., Pietrobon, D., Balbi, A., Baldi, P., Cabella, P., Kerkyacharian, G., Natoli, P., Picard, D., \& Vittorio, N. 2008, MNRAS, 383, 539

Narcowich, F. J., Petrushev, P., \& Ward, J. D. 2006, SIAM J. Math. Anal., 38, 574

Planck Collaboration 2013, ArXiv e-prints arxiv:1303.5084

Regan, D., Gosenca M., Seery D. 2013, ArXiv e-prints arxiv:1310.8617

Sekiguchi, T., Sugiyama, N. 2013, JCAP, 09, 2

Troja, A. et al. 2014, Manuscript in preparation

Wick, G. C. 1950, Physical Review, 80, 268 